\title{
Set-and Code-Specific Activation in the Frontal Cortex: An fMRI Study of Encoding and Retrieval of Faces and Words
}

\section{Citation}

McDermott, Kathleen B., Randy L. Buckner, Steven E. Petersen, William M. Kelley, and Amy L. Sanders. 1999. "Set-and Code-Specific Activation in the Frontal Cortex: An fMRI Study of Encoding and Retrieval of Faces and Words." Journal of Cognitive Neuroscience 11 (6) (November): 631-640. doi:10.1162/089892999563698.

\section{Published Version}

doi:10.1162/089892999563698

\section{Permanent link}

http://nrs.harvard.edu/urn-3:HUL.InstRepos:33896769

\section{Terms of Use}

This article was downloaded from Harvard University's DASH repository, and is made available under the terms and conditions applicable to Other Posted Material, as set forth at http:// nrs.harvard.edu/urn-3:HUL.InstRepos:dash.current.terms-of-use\#LAA

\section{Share Your Story}

The Harvard community has made this article openly available.

Please share how this access benefits you. Submit a story.

Accessibility 


\title{
Set- and Code-Specific Activation in the Frontal Cortex: An fMRI Study of Encoding and Retrieval of Faces and Words
}

\author{
Kathleen B. McDermott, Randy L. Buckner, Steven E. Petersen, \\ William M. Kelley, and Amy L. Sanders \\ Washington University and Washington University School of Medicine
}

\begin{abstract}
The frontal cortex has been described as playing both "setspecific" and "code-specific" roles in human memory processing. Set specificity refers to the finding of goal-oriented differences in activation patterns (e.g., encoding relative to retrieval). Code specificity refers to the finding of different patterns of activation for different types of stimuli (e.g., verbal/nonverbal). Using a two (code: verbal, nonverbal) by two (set: encoding, retrieval) within-subjects design and fMRI, we explored the influence of type of code and mental set in two regions in the frontal cortex that have been previously shown to be involved in memory. A region in the dorsal extent of the
\end{abstract}

\section{INTRODUCTION}

Recent neuroimaging studies have drawn increasing attention to the idea that frontal cortical areas may contribute to human memory; however, the nature of the contributions of these areas is incompletely understood (see Buckner, 1996; Cabeza \& Nyberg, 1997; Desgranges, Baron, \& Eustache, 1998; Fletcher, Frith, \& Rugg, 1997, for reviews). The goal of understanding how the frontal cortex contributes to memory is elusive at least in part because the frontal lobes represent a broad subdivision of the cortex, which comprises a set of heterogeneous areas that may not link to memory processes in any simple manner.

An examination of the patterns of activations reported across the literature suggests that there may be both code- and set-specific effects in the frontal cortex; further, these effects appear to occur in distinct frontal regions. The verbal/nonverbal nature of the materials being processed (and by inference the verbal/nonverbal nature of the codes subjects invoke for processing) seems to drive the laterality of activation in at least one region of the frontal cortex: the inferior frontal gyrus at or near the boundary of BA $6 / 44$. Hence, we can think of this region as exhibiting code specificity. Another region, more anteriorly placed, seems to exhibit sensitivity to whether or not subjects are attempting to retrieve inferior frontal gyrus (BA 6/44) demonstrated code-specific effects. Specifically, an interaction of material type with hemisphere was obtained, such that words produced predominantly left-lateralized activation, whereas unfamiliar faces elicited predominantly right-lateralized activation. A region of the right frontal polar cortex (in or near BA 10), which has been activated in many memory retrieval studies, showed set-specific activation in that it was more active during retrieval than encoding. These data demonstrate that distinct regions in the frontal cortex contribute in systematic yet different ways to human memory processing. previous experiences (i.e., their mental set). The primary goal of the present experiment was to determine whether both code-specific (verbal/nonverbal) and setspecific (encoding/retrieval) activation patterns would be manifested in distinct regions of the frontal cortex, within a single group of subjects.

\section{Code Specificity: Inferior Frontal Gyrus}

The idea that the encoding and retrieval of different types of materials engage different brain regions has its origins in the neuropsychological literature (e.g., Gazzaniga, Bogen, \& Sperry, 1962; Gazzaniga \& Smylie, 1983; Milner, 1971). Patients with lesions in the left hemisphere tend to have difficulty processing verbal information, whereas those with lesions in the right hemisphere tend to have difficulty processing nonverbal information. Hemispheric asymmetries are found in normal subjects also: Verbal information is processed more efficiently when flashed to the right hemifield (left hemisphere) than to the left hemifield; conversely, spatially coded information is processed more efficiently when presented to the left hemifield than to the right (Klatzky \& Atkinson, 1971).

Thus, different materials are thought to engage different types of codes, and the different codes are probably processed by brain areas that show different patterns 
of lateralization. Recent neuroimaging results have refined this hypothesis by identifying specific brain regions that are sensitive to the verbal/nonverbal nature of the stimuli (Kelley, Miezin, et al., 1998; Kelley et al., 1999; Wagner, Desmond, Glover, \& Gabrieli, 1998; Wagner, Poldrack, et al., 1998; but see Grady et al., 1995; Haxby et al., 1996).

For example, Kelley, Miezin, et al. (1998) demonstrated that when people intentionally encoded words for a later memory task, a set of regions, including the posterior frontal cortex in the left inferior frontal gyrus (BA 6/44), were robustly active. When the subjects studied unfamiliar faces, however, activity in a similar region was right-lateralized. Further, when subjects encoded pictures of line-drawn objects, the inferior frontal cortex was active bilaterally. These researchers explained their results by appealing to the differences in verbal processing encouraged by the materials. That is, words tend to elicit verbal processing (and therefore left-lateralized activity); unfamiliar faces, which have no obvious verbal referents, tend to elicit nonverbal processing (and therefore right-lateralized activity); finally, line-drawn objects that can be named (e.g., a drawing of a frog) afford both nonverbal and verbal processing codes (and therefore lead to bilateral activation patterns). Wagner, Poldrack, et al. (1998) reported a similar pattern of results in that encoding and retrieval of words led to greater activity in the left inferior frontal gyrus than did texture patterns, and texture patterns elicited greater activation levels than did words in a homologous region on the right. Further evidence that verbal processing is the relevant dimension driving face/word differences comes from the finding that encoding of famous faces, which, like line-drawn objects, can be easily labeled verbally, produces bilateral activation (much like the line drawings, Kelley et al., 1999).

In addition to demonstrating material specificity in inferior frontal regions, these studies have also suggested that the inferior frontal cortex plays an important role in memory encoding (for review see Buckner, Kelley, \& Petersen, 1999). For example, Kelley, Miezin, et al. (1998) examined activation in this region when subjects were instructed simply to look at the stimuli; these instructions led to poor performance on a later recognition memory test as well as diminished activity in the inferior frontal gyrus. Other neuroimaging studies have also contrasted tasks that facilitate subsequent retrieval with those that lead to poor subsequent retrieval and have noted similar modulation of frontal activity. For example, attending to the meaning of words (e.g., deciding whether a word is abstract or concrete) enhances later memory performance (the level-of-processing effect, Craik \& Lockhart, 1972) and elicits greater activity in the left inferior frontal gyrus (Demb et al., 1995; Kapur et al., 1994) relative to performing surface-level processing of words (e.g., counting the number of vowels in a word). Perhaps the most compelling evidence thus far that the frontal cortex, specifically the region within the posterior portion of the inferior frontal gyrus, is linked strongly to encoding is that activation levels in this region have been shown to be predictive of whether or not an item is subsequently retrieved on a recognition memory test (Brewer, Zhao, Desmond, Glover, \& Gabrieli, 1998; Wagner, Schacter, et al., 1998). Moreover, this predictive power has been shown both for verbal materials (in the left inferior frontal gyrus, Wagner, Schacter, et al., 1998) and pictorial materials (in the right inferior frontal gyrus, Brewer et al.).

A final argument for the position that the inferior frontal gyrus contributes to encoding is grounded in dual coding theory (Paivio, 1971), which posits that the reason pictures are typically better remembered than words (the picture superiority effect) is that pictures are coded both imaginally and verbally. As discussed above, pictures elicited bilateral activation in the inferior frontal gyrus, whereas words elicited left-lateralized activation in Kelley, Miezin, et al.'s (1998) study. Thus, it seems reasonable to argue that the source of later differences in retrieval success is mediated in part by bilateral activity in the inferior frontal gyrus during encoding.

However, it is also reasonable to expect that activation in the inferior frontal gyrus is not limited just to encoding. For recognition memory to be successful, subjects must perform many of the same cognitive operations that are engaged during encoding (e.g., visual deciphering of the stimulus and access to its meaning). That is, active, effortful processing may lead to activity in these regions across a wide range of task goals (e.g., encoding and retrieval), all of which can encourage code-specific representations of the information. The open question, then, is whether code-specific asymmetries in the inferior frontal gyrus generalize across task goals to retrieval. Specifically, we were interested in whether hemispheric asymmetries for different types of materials would be observed on a recognition memory test in which subjects were shown words and faces and asked whether or not they had previously studied the items. Our hypothesis was that retrieval would show laterality effects similar to those seen at encoding: Verbal processing would be left-lateralized, and nonverbal processing rightlateralized. However, predictions regarding the effects of mental set (encoding or retrieval) were much less decisive. On the basis of the neuroimaging literature showing the predictive power of activation in this region for later memory performance, it could be argued that we would observe a main effect of encoding over retrieval. On the basis of the logic presented above, however, it seemed equally plausible that we would see no set effects in this region. That is, active verbal or nonverbal processing, regardless of whether it involved an encoding or retrieval task, would elicit robust activation of the inferior frontal gyrus.

The current study was designed to pull apart these possibilities by using a two (materials: words, unfamiliar 
faces) by two (set: encoding, retrieval) within-subject factorial design. The encoding of words and unfamiliar faces were replications of conditions reported by Kelley, Miezin, et al. (1998). Therefore, we expected to replicate their finding of a crossover interaction: Words would produce left-lateralized activation, whereas faces would produce right-lateralized activation. These laterality effects would presumably arise from the verbal/nonverbal processing demands afforded by the materials (and not the visual features of the materials themselves). The question was whether activations observed during retrieval would mimic those seen at encoding; we expected that they would. That is, recognition memory for words would elicit left-lateralized activation, whereas recognition memory for faces would elicit right-lateralized activation in inferior frontal gyrus.

\section{Set Specificity: Frontal Polar Cortex}

A right-lateralized region of the frontal polar cortex (at or near BA 10) has been shown to be active across a variety of episodic retrieval tasks (Andreasen et al., 1995; Buckner, Koutstaal, Schacter, Wagner, \& Rosen, 1998b; Buckner et al., 1995, 1996, 1998a; Fletcher et al., 1995; Haxby et al., 1996; Rugg, Fletcher, Frith, Frackowiak, \& Dolan, 1996; Schacter, Alpert, Savage, Rauch, \& Albert, 1996; Schacter, Buckner, Koutstaal, Dale, \& Rosen, 1997; Squire et al., 1992; Tulving, Kapur, Craik, Markowitsch, \& Houle, 1994; for reviews see Buckner, 1996; Cabeza \& Nyberg, 1997). This region has been argued by some to represent the "set" or goal of trying to retrieve past experiences (Buckner et al., 1998a; McDermott et al., in press; Nyberg, Cabeza, \& Tulving, 1996; Tulving et al., 1994; Wagner, Desmond, et al., 1998), although the exact function of the region is still controversial (MacLeod, Buckner, Miezin, Petersen, \& Raichle, 1998; Nolde, Johnson, \& Raye, 1998; Rugg et al., 1996; Schacter et al., 1996). It should be noted that Tulving and colleagues (see Nyberg et al., 1996; Tulving et al., 1994) proposed a broad heuristic with respect to the role of the frontal cortex in human memory: Encoding of information draws upon the left frontal cortex more than the right frontal cortex, whereas episodic retrieval draws upon the right frontal cortex (more than left). This description, called the Hemispheric Encoding/Retrieval Asymmetry (HERA) model, was initially applied to only verbal materials (Tulving et al., 1994) but was then extended to also apply to nonverbal materials (Nyberg et al., 1996). Although the utility of this broad distinction is debatable, there is wide acceptance that there is at least one rightlateralized region (i.e., the frontal polar cortex) that seems to be engaged preferentially during retrieval, relative to encoding.

The goal of the present experiment with respect to the right frontal polar cortex was to explore whether this region would be sensitive to the encoding/retrieval variable (i.e., set) but insensitive to the type of codes elicited by the stimuli. That is, we expected to see a main effect of set (retrieval > encoding) but no main effect of (or interaction with) material type. The conjoint result of code-specific effects (as discussed above, for one region of the frontal cortex) along with set-specific effects (in a separate region of the frontal cortex) would provide a powerful functional dissociation and may help resolve the complex relation between frontal regions and processing operations engaged during human memory task performance. ${ }^{1}$

\section{RESULTS AND DISCUSSION}

\section{Behavioral}

Behavioral data for three subjects were lost due to equipment difficulties with the fiber-optic keypress. The eight remaining subjects showed reasonably good accuracy for both the faces and words; mean hit rates were 0.79 and 0.80 for faces and words and mean false alarm rates were 0.28 and 0.20 for faces and words, respectively. From these data, we can conclude that subjects showed good discrimination between old and new items in both conditions.

\section{Functional Magnetic Resonance Imaging (fMRI)}

\section{Whole Brain Activations}

Although the focus in the present study was on two specific regions of the frontal cortex, we note that there is a complex network of activity underlying performance of these tasks, and these networks are highlighted in Figure 1. Panel 1 of this figure shows activity for all conditions (combined) across all 11 subjects. This panel represents the statistical image from which peak points of activation were identified. These activations reflect both the visual and motor components of the task as well as higher-level activations (e.g., the two regions targeted: the inferior frontal gyrus and frontal polar cortex as well as several bilateral parietal regions).

\section{Inferior Frontal Gyrus}

Figure 1 (panel 1, second slice) shows activation maps through the peak activation points $(-43,5,28$ and $45,1,32$ for the left and right inferior frontal gyri, respectively). Figure 2 displays these data in terms of mean percentage of signal change for the four conditions. A 2 (hemisphere) $\times 2$ (materials) $\times 2$ (task) repeated-measures analysis of variance (ANOVA) on the magnitudes demonstrated a single main effect (for materials, faces $>$ words, $F(1,10)=29.5, p<0.001)$ and several interactions: The interactions between hemisphere and material, $F(1,10)=36.24, p<0.0001$, and between hemisphere and set, $F(1,10)=9.71, p<0.01$, were significant, as was the three-way interaction, $F(1,10)=5.57, p<0.04$. This three-way interaction can be conceptualized by examin- 
Figure 1. Panel 1. Statistical map demonstrating activity across the whole brain for the composite image (from all conditions for all 11 subjects $^{2}$ ). Colored pixels exceeded the statistical threshold of $p<$ 0.05 and were superimposed on the corresponding anatomical images. The color scale represents the significance level for activations from red (lowest) to yellow (highest). The left side of the images corresponds to the left side of the brain. Regions shown include the left motor cortex (A: $-39,-27,50$; BA 3/4), supplementary motor area $(\mathrm{B}$ : $-3,11,50$; BA 6), bilateral superior and inferior parietal cortex/precuneus (C, D, E, F: $-27,-65,46 ; 27,-65,50$; $-27,-61,38 ; 25,-61,40$; BA $7,19 / 39)$, bilateral posterior inferior frontal gyrus $(\mathrm{G}, \mathrm{H}$ : $-43,5,28 ; 45,1,32$; BA 6/44), right frontal polar cortex (I: $39,55,2 ; \mathrm{BA} 10$ ), bilateral fron tal opercula (J, K: $-29,21,2$; 29,19,4; BA 47/45), bilateral visual cortex (L, M: multiple peaks), and bilateral fusiform gyrus (N, O: multiple peaks). Panels 2 and 3. Statistical map demonstrating code-specificity effects during encoding (panel 2) and retrieval (panel 3) across the whole brain $(p<0.05)$. Regions more active in word processing (relative to face processing) are shown on a blue-to-green color scale; those more active in face processing (relative to word processing) are shown on a red-to-yellow color scale. Regions more active for words than faces include the left superior frontal cortex (A), left posterior inferior frontal gyrus (B), left parietal cortex (C), left ventral inferior frontal cortex (D), and left lateral temporal cortex (E). Regions more active for faces than words include the right parietal cortex $(F, G)$, right posterior inferior frontal gyrus $(\mathrm{H})$, bilateral visual cortex (I, J), and bilateral fusiform gyrus $(\mathrm{K}, \mathrm{L})$.

ing the hemisphere by material interaction at the two levels of set. The interaction of hemisphere by material was reliable for both encoding, $F(1,10)=38.35, p<$ 0.0001 , and retrieval, $F(1,10)=24.49, p<0.001$. An analysis of the simple effects demonstrated that for words, activation was greater on the left than the right for both encoding, $t(10)=4.80, p<0.0004$, and retrieval, $t(10)=2.02, p<0.036$. Conversely, for faces, activation was greater on the right than the left for both encoding, $t(10)=3.69, p<0.002$, and retrieval, $t(10)=3.63, p<$
0.002 . The three-way interaction can be understood as showing that the laterality effect for words was more pronounced at encoding than retrieval, whereas the laterality effect for faces was greater for retrieval than encoding. In summary, systematic laterality effects were obtained for both encoding and retrieval; words produced left-lateralized activations, whereas faces elicited right-lateralized activity. 
Figure 2. Mean magnitudes (in percent signal change of activation) for a sphere (12$\mathrm{mm}$ radius, significantly active voxels only) surrounding activation peaks in the left and right inferior frontal gyri. Error bars represent the standard error of the mean.

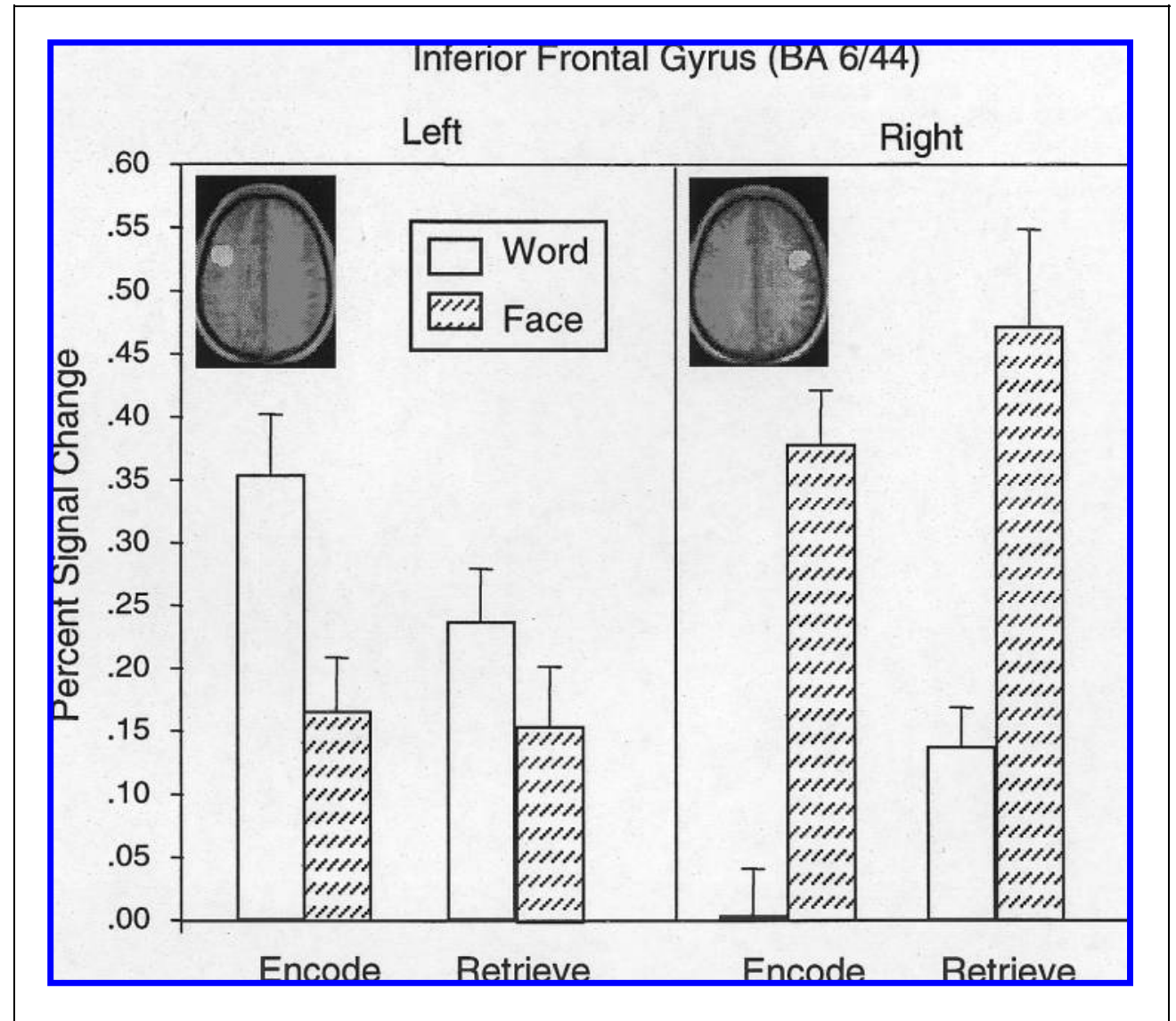

\section{Right Frontal Polar Cortex}

Panel 1 of Figure 1 (third slice) shows statistical activation maps for all conditions combined in the right frontal polar cortex (peak 39,55,2). Figure 3 shows the magnitude of signal change shown for each of the four conditions for the reliable points within a 12-mm sphere around this region. The differences in encoding and retrieval can be seen, with mean magnitudes of 0.22 and 0.42 for encoding and retrieval, respectively. A 2 (set: encoding, retrieval) $\times 2$ (material: faces, words) repeated measures ANOVA on the magnitudes demonstrated a single reliable effect: a main effect for set, $F(1,10)=4.99$, $p<0.05$. Thus, as predicted, retrieval activated the right frontal polar cortex more than did encoding. Although no interaction with material type was present, we performed planned comparisons between encoding and retrieval for both words, $t<1$, and faces, $t(10)=3.02$, $p<0.006$. Although the effect for words was not reliable, the numerical direction of the effect was in the predicted direction ( $M=0.27$ encoding, 0.34 retrieval), and this effect has been reported numerous times in the literature (see Buckner, 1996 and Cabeza \& Nyberg, 1997 for reviews); we therefore endorse the conclusion that the frontal polar cortex is more active for retrieval than encoding for both words and faces.

\section{Whole-Brain Differences in Face and Word} Processing: Exploratory Analyses

Panels 2 and 3 of Figure 1 show regions preferentially active for words (blue-to-green color scale) and faces (red-to-yellow color scale). These panels demonstrate that although the predicted code-specific effects occurred in the inferior frontal gyrus, these effects are not localized to the region of interest in the current study. For current purposes, the two panels, representing encoding and retrieval, can be viewed as independent replications of each other in that the overall network of activity underlying encoding and retrieval were highly similar, although subtle differences can be seen. Verbal processing activated the left inferior frontal gyrus to a greater extent than did faces, but it also preferentially activated a network of left-lateralized regions; these regions include the left superior frontal, a left lateral parietal region, the left temporal cortex, and the left ventral frontal cortex. Conversely, the processing elicited by the unfamiliar faces activated the right inferior frontal gyrus to a greater extent than words, but it also preferentially activated other right-lateralized regions. In addition, bilateral visual cortical regions and the fusiform gyrus were more active during face processing than word processing. The parietal cortex tentatively appears to have multiple medial and lateral regions that behave differently 


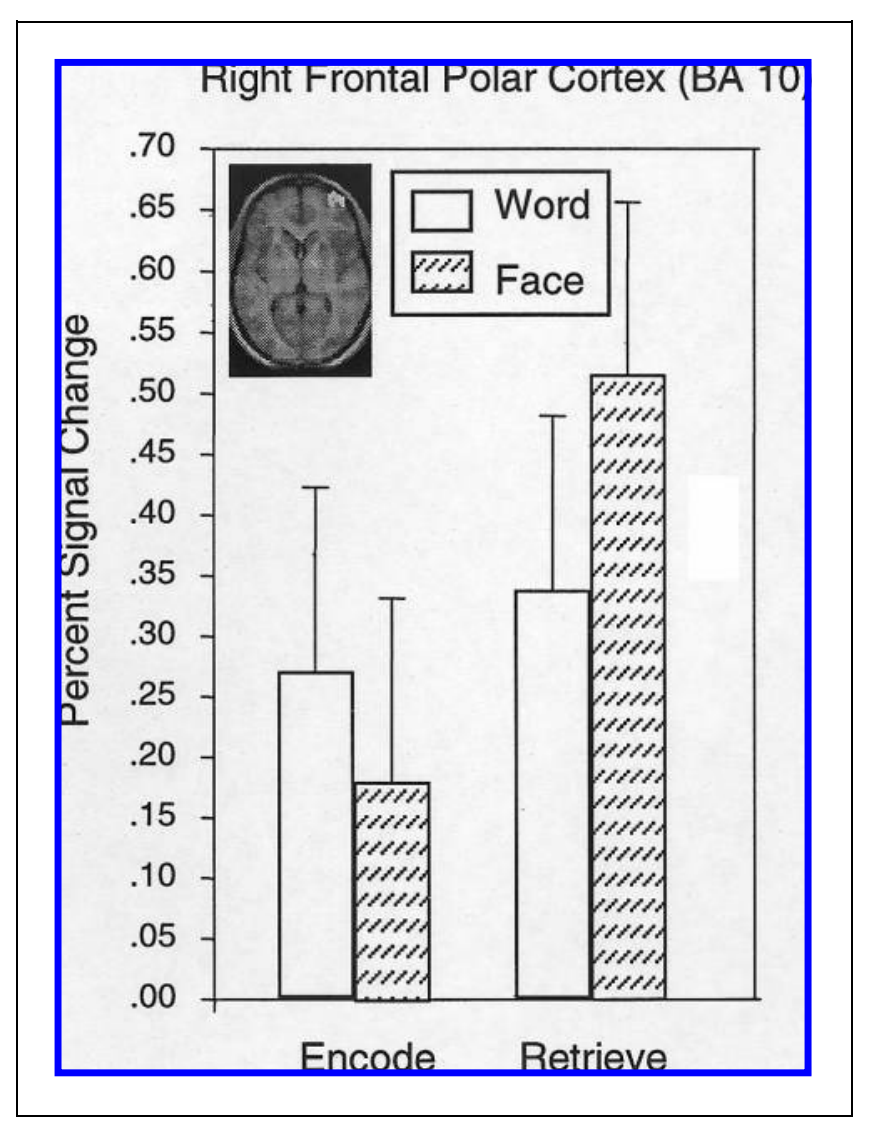

Figure 3. Mean magnitudes (in percent signal change of activation) for a sphere (12-mm radius, significantly active voxels only) surrounding the activation peak in the right frontal polar cortex. Error bars represent the standard error of the mean.

from one another. Full understanding of these additional regions will have to await future studies.

\section{GENERAL DISCUSSION}

We have shown that two distinct regions of the frontal cortex contribute in different but systematic ways to episodic memory tasks. The inferior frontal gyrus demonstrated code-specific effects. The right frontal polar cortex demonstrated set-specific effects. These findings are consistent with the prior literature; moreover, they advance our understanding of the role of the frontal cortex in human memory function in several ways. First, they show that the hemispheric laterality effects with respect to verbal/nonverbal stimuli are not restricted to encoding but extend to retrieval as well. That is, processing of verbal and nonverbal information, regardless of whether an episodic encoding or retrieval task is performed, yields systematic asymmetries in the inferior frontal gyrus. Second, the functional dissociation of two regions of the frontal cortex (the bilateral inferior frontal gyrus and right frontal polar cortex) with respect to memory provides an empirical demonstration of the point that different regions of the frontal cortex play different yet systematic roles in human memory processing (see Buckner, 1996; Petrides, Alivisatos, \& Evans, 1995, for discussion of this point). Third, exploratory analyses demonstrated that laterality effects for verbal/nonverbal information are not restricted to the regions of interest in this report but extend to other regions of cortex as well.

The extension of the verbal/nonverbal asymmetries from encoding to retrieval is a natural one in light of the memory literature at large. Episodic encoding, semantic retrieval, and episodic retrieval are intricately intertwined: Encoding of information can involve both semantic and episodic retrieval; likewise, episodic retrieval typically involves semantic retrieval and often leads to encoding of information (Tulving, 1983). In this light, it is not surprising that activation patterns are generally similar across the tasks.

With the present results in mind, one could still argue that the inferior frontal gyrus region activated in the present study plays a special role in encoding; however, we feel that this perspective may be missing a deeper point. As noted, episodic encoding, semantic retrieval, and episodic retrieval all activate the inferior frontal gyrus and share similar processing demands related to elaboration of verbal materials (in the instance of left inferior frontal gyrus activation) or nonverbal materials (in the instance of right inferior frontal gyrus activation). The elaborative processing invoked by these tasks can result in encoding. By this view, certain regions within the inferior frontal gyrus are relevant to the code-specific processing of information, which may also begin a cascade of events that sends information to other brain regions and eventually leads to the formation of a memory trace (Buckner et al., 1999). Retrieval tasks may give rise to this activation because they demand elaboration upon code-specific information; an end product of this processing may be incidental encoding. Indeed, there is a large literature demonstrating the facilitating effects of an initial retrieval test upon subsequent retrieval of an event (see Glover, 1989, for a review). Nevertheless, the inferior frontal gyrus does participate robustly in retrieval tasks and shows laterality effects during retrieval similar to those seen at encoding, as shown in the present study and by Wagner, Poldrack, et al. (1998).

The implications for HERA of the recent hemispheric asymmetries for verbal and nonverbal information have been debated (Kelley, Buckner, \& Petersen, 1998; Nyberg, Cabeza, \& Tulving, 1998; Poldrack \& Gabrieli, 1998). Kelley, Miezin, et al. (1998) pointed out that a strong version of HERA as extended to nonverbal materials would not have predicted right-lateralized activity in the frontal cortex during face encoding. The present dataset replicates this pattern and demonstrates a further complication (i.e., left-lateralized activation during retrieval of words).

The findings with respect to the frontal polar cortex, however, are consistent with the HERA postulates. That 
is, the mental set invoked when performing a retrieval task activates the right frontal polar cortex rather ubiquitously (see Buckner, 1996, for a review), and whether people are successful or not during this retrieval attempt does not always seem to drive activation here (see Buckner et al., 1998a; Kapur et al., 1995; Nyberg et al., 1995; Wagner, Desmond, et al., 1998; but see Rugg et al., 1996, for an alternative interpretation). This dataset complements those in the literature by providing a withinsubjects demonstration that the set-specific pattern seen in the frontal polar cortex is dissociable from another memory-related region in the frontal cortex.

Exploratory analyses further showed that for regions in which lateralized activity was observed, words produced predominantly left-lateralized activation, whereas the unfamiliar faces led to predominantly right-lateralized activation. Thus, the asymmetries focused on with respect to the inferior frontal gyrus may extend to other regions. Therefore, although the inferior frontal gyrus tends to show a specific pattern of laterality effects, it does not seem to be the sole region demonstrating such effects. We have targeted the inferior frontal gyrus in this study, but a full understanding of laterality effects will come only after the entire network has been more fully explored.

Similarly, the full story with respect to the codes underlying the asymmetries occurring in the inferior frontal gyrus has not yet been worked out. We have chosen to adopt the verbal/nonverbal heuristic here because that description has been common in the neuropsychological literature and seems to capture the current findings and predict other findings (e.g., with respect to unfamiliar and famous faces) reasonably well. However, this description will surely be shown to be incomplete, for several reasons. First, verbal and nonverbal processing are not represented by a single continuum: Variables can selectively increase verbal processing (and activation in left inferior frontal gyrus) without decreasing the level of nonverbal processing (Kelley et al., 1998). Second, there are many aspects to "verbal" processing (e.g., phonological, lexical, semantic, associative), and precisely which of these component processes contribute to activations in this region has not been fully described. There is evidence for the importance of semantics (Wagner, Schacter, et al., 1998) but also evidence that phonological codes are important (Awh et al., 1996). Even more problematic is the "nonverbal" category, which at this point is something of a leftover label. There has been some speculation in the lesion literature about the specific types of processing involved here (see Sergent, 1983), but, again, a precise characterization has not yet emerged. Nevertheless, the distinction has been useful at a broad level in making predictions about levels of activation in the inferior frontal cortex.

One final caveat is that we have discussed the right frontal polar cortex as a retrieval region. This characterization is accurate in that the region does tend to be more active during episodic retrieval than encoding across both verbal and nonverbal materials (Cabeza \& Nyberg, 1997). However, this region may not be exclusively a "retrieval" region; activation of this region has been seen in working memory tasks, as well (see MacLeod et al., 1998, for an overview). It remains to be seen whether there are common features of these tasks that lead to similar activation patterns in the frontal polar cortex.

In summary, we have used a within-subjects design to demonstrate a dissociation between two distinct regions of the frontal cortex with respect to their roles in human memory function. The inferior frontal gyrus is invoked during encoding and retrieval of verbal and nonverbal information. Moreover, activation in this region is left-lateralized for verbal processing and is right-lateralized for nonverbal processing. A second region (the right frontal polar cortex) seems to be invoked preferentially during episodic retrieval. These results are consistent with our understanding of the role of the frontal cortex in human memory and also advance that understanding by projecting a broader picture than was previously available.

\section{METHOD}

\section{Subjects}

Twelve subjects (six males, six females, mean age 22.6, range 18 to 30 years) were recruited from the Washington University community in return for payment. All were right-handed, native speakers of English, had normal or corrected-to-normal vision, and reported no history of significant neurological problems. Subjects provided informed consent in accordance with the guidelines set by the Washington University Human Studies Committee.

\section{Design and Materials}

A 2 (set: encoding, retrieval) $\times 2$ (materials: faces, words) within-subjects design was used. Words $(N=192)$ were the names of concrete objects; they were drawn from the Snodgrass and Vanderwart (1980) norms. Faces $(N=$ 192, one-half male, one-half female) were color pictures of nonfamous, unfamiliar people cut from magazines and digitized.

Counterbalancing for each stimulus type was achieved by dividing 192 items into three sets (of 64 items); each set occurred in each condition (studied, nonstudied but tested, or neither studied nor tested) an equal number of times across subjects.

\section{Procedure}

Scans were conducted on a Siemens 1.5 Tesla Vision System (Erlangen, Germany) with a standard circularly polarized head coil. A Power Macintosh computer (Ap- 
ple, Cupertino, CA) and Psyscope software (Cohen MacWhinney, Flatt, \& Provost, 1993) displayed the visual stimuli. Subjects responded during the retrieval tests by pushing one of two keys on a fiber-optic light-sensitive keypress connected to a Psyscope Button Box (Carnegie Mellon University, Pittsburgh, PA). An LCD projector (Sharp, model XGE850) projected stimuli onto a screen placed at the head of the bore. Subjects viewed the screen via a mirror fastened to the head coil. A pillow and thermoplastic face mask were used to minimize head movement. Headphones dampened scanner noise and enabled communication with subjects.

Structural images were acquired using the MPRAGE sequence with $2-\mathrm{mm}$ isotropic voxels. Functional images were collected with an asymmetric spin-echo-planar sequence sensitive to blood oxygenation level-dependent (BOLD) contrast ( $\mathrm{TR}=2.68-\mathrm{sec}, 3.75-\times 3.75-\mathrm{mm}$ in-plane resolution). In each functional run, 102 sets of 16 contiguous, 8-mm-thick axial images were acquired parallel to the anterior-posterior commissure plane; this procedure offered whole-brain coverage at a high signal-tonoise ratio (Conturo et al., 1996). Each run lasted approximately $41 / 2 \mathrm{~min}$ (2.68 sec per whole-brain acquisition, with 102 such acquisitions per run). There were approximately $3 \mathrm{~min}$ between runs, during which time subjects were permitted to rest.

Each subject participated in two encoding runs (one containing words and the other faces), followed by two recognition runs (one run corresponding to the previously studied words and the other to faces); they then cycled through the same sequence (two encoding runs, two recognition runs) with different stimuli and a different order (i.e., faces were studied first if words had been studied first in the previous cycle). Thus, each subject participated in eight runs, divided equally among the four conditions.

A blocked design was used for both encoding and retrieval runs. There were seven blocks per run; four of the blocks were "task" blocks (during which subjects encoded or retrieved faces or words), and three of the blocks were "fixation" blocks (during which subjects looked at a fixation point and remained as still as possible). Task and fixation blocks alternated. Task blocks lasted $42.88 \mathrm{sec}$ (2-sec stimulus duration, 680-msec interstimulus interval, 16 stimuli per block). Each (2680 msec) trial began at the onset of the TR (or repetition time, the time required for one whole-brain scan). Therefore, one task trial occurred during each TR. Each fixation block lasted 10 TRs, or 26.8 sec.

Although a blocked design was used, trials in the recognition runs occurred in a fixed random order. That is, half of the items tested had been studied previously, and half had not, but the ordering of old/new trials within each run was unpredictable.

During the encoding phase, subjects were instructed to pay close attention to the stimuli (words or faces) and to remember as many as possible for a later recognition memory test; these instructions were adopted because they gave rise to robust laterality effects in Kelley, Miezin, et al.,'s (1998) study, although studies by Brewer et al. (1998) and Wagner, Schacter, et al. (1998) suggest that these hemispheric asymmetries generalize across a variety of encoding instructions and across intentional/incidental encoding. During the test phase, subjects were given identical presentation parameters but had to respond to each item to indicate whether it had been previously studied or not. We built this difference into the design to maximize our chances of obtaining both inferior frontal activation (which has typically occurred under no- response conditions) and frontal polar activation (which has typically occurred under response conditions, due to the nature of retrieval tests). During the retrieval test, subjects were instructed to respond while the stimulus was on the screen. Subjects were told that during the fixation phase they should look at the fixation point while remaining still.

\section{Data Analysis}

Data for each subject were subjected to the processing stream developed at Washington University, which includes movement-correction within and across runs using an automated procedure (Snyder, 1996) and whole brain normalization to a common mode of 1000 to allow for comparisons across subjects (see Ojemann et al., 1997, for an overview).

The data were analyzed using an implementation of the general linear model. First we identified regions active in separate condition types (e.g., encoding words relative to fixation). Specifically, we cross-correlated the time course of the BOLD response to each condition at each voxel. The resulting magnitudes were then used to compute $z$ statistics to determine if they differed reliably from 0.00 (Friston, Jezzard, \& Turner, 1994; Worsley \& Friston, 1995). In addition, comparisons between conditions were computed by assigning appropriate weights to each condition. This approach allowed for direct comparisons to determine regions differentially active in one condition relative to another (e.g., faces to words).

The resulting statistical images were warped into standardized atlas space (Talairach \& Tournoux, 1988) and averaged across subjects. Unbiased peak regions of interest were obtained by creating an average statistical image of all four conditions (encoding of words, encoding of faces, retrieval of words, retrieval of faces). The resulting composite image was then corrected for multiple comparisons (Ollinger, 1997) such that $p<0.05$, where $p$ refers to the probability of a single erroneous activation in the image volume. The correction method uses a region-size-dependent threshold to guarantee that the experiment-wide Type I error is $<0.05$. An automated peak-search algorithm (Mintun, Fox, \& Raichle, 1989) identified the location (in atlas coordinates) of peak activations on the basis of level of statistical significance 
and cluster size. After identification of the peaks for bilateral posterior inferior frontal gyrus and right anterior frontal regions, mean magnitudes (in percent change) for each subject for all significant voxels (as defined on the group average image) within a $12-\mathrm{mm}$ radius of the peak points were obtained. ${ }^{2}$

ANOVA was used to reliably identify differences in the two regions of interest. For posterior inferior frontal regions, a 2 (hemisphere: left, right) $\times 2$ (task: encoding, retrieval) $\times 2$ (materials: faces, words) ANOVA was computed. For the right frontal polar region, a 2 (task: encoding, retrieval) $\times 2$ (materials: faces, words) ANOVA was computed.

\section{Acknowledgments}

Supported by NS32979 to SEP and MH57506 to RLB. We are grateful to Dave Balota, David Donaldson, Roddy Roediger, Endel Tulving, and Anthony Wagner for providing comments on an earlier draft of the paper and to Neal Cohen for making his set of face stimuli available to us.

Reprint requests should be sent to Kathleen McDermott, Division of Radiological Sciences, Box 8225, Washington University School of Medicine, 4525 Scott Avenue, St. Louis, MO 63110, or via e-mail: kmcd@npg.wustl.edu.

\section{Notes}

1. Our interest in this study centered on two specific regions in the frontal cortex because the recent literature has suggested that these two regions play important roles in human memory processing. However, we do not mean to imply that code- or set-specific activity occurs in these regions exclusively; similar patterns may be uncovered in other regions (in the frontal cortex or elsewhere). However, the two regions of interest in the present study were chosen because they seemed on the basis of the literature to be likely candidates to demonstrate functional dissociations.

2. At this stage, data for 1 of the 12 subjects were discarded because the magnitudes for this subject were extreme outliers in all four conditions. Peak points were then redefined (on the basis of 11 subjects only), and all subsequent analyses are based upon 11 subjects.

\section{REFERENCES}

Andreasen, N. C., O'Leary, D. S., Arndt, S., Cizadlo, T., Hurtig, R., Rezai, K., Watkins, G. L., Ponto, L. L. B., \& Hichwa, R. D. (1995). Short-term and long-term verbal memory: A positron emission tomography study. Proceedings of the $\mathrm{Na}$ tional Academy of Sciences, USA, 92, 5111-5115.

Awh, E., Jonides, J., Smith, E. E., Schumacher, E. H., Koeppe, R. A., \& Katz, S. (1996). Dissociation of storage and rehearsal in verbal working memory: Evidence from positron emission tomography. Psychological Science, 7, 25-31.

Brewer, J. B., Zhao, A., Desmond, J. E., Glover, G., \& Gabrieli, J. D. E. (1998). Making memories: Brain activity that predicts how well visual experience will be remembered. $\underline{S c i-}$ ence, 281, 1185-1187.

Buckner, R. L. (1996). Beyond HERA: Contributions of specific prefrontal brain areas to long-term memory retrieval. Psychonomic Bulletin \& Review, 3, 149-158.

Buckner, R. L., Kelley, W. M., \& Petersen, S. E. (1999). Frontal cortex contributes to human memory formation. Nature Neuroscience, 2, 311-314.

Buckner, R. L., Koutstaal, W., Schacter, D. L., Dale, A. M., Rotte, M., \& Rosen, B. R. (1998a). Functional-anatomic study of episodic retrieval: II. Selective averaging of event-related fMRI trials to test the retrieval success hypothesis. Neuroimage, 7, 163-175.

Buckner, R. L., Koutstaal, W., Schacter, D. L., Wagner, A. D., \& Rosen, B. R. (1998b). Functional-anatomic study of episodic retrieval using fMRI: I. Retrieval effort versus retrieval success. Neuroimage, 7, 151-162.

Buckner, R. L., Petersen, S. E., Ojemann, J. G., Miezin, F. M., Squire, L. R., \& Raichle, M. E. (1995). Functional anatomical studies of explicit and implicit memory retrieval tasks. Journal of Neuroscience, 15, 12-29.

Buckner, R. L., Raichle, M. E., Miezin, F. M., \& Petersen, S. E. (1996). Functional anatomic studies of memory retrieval for auditory words and visual pictures. Journal of Neuroscience, 16, 6219-6235.

Cabeza, R., \& Nyberg, L. (1997). Imaging cognition: An empirical review of PET studies with normal subjects. Journal of Cognitive Neuroscience, 9, 1-26.

Cohen, J. D., MacWhinney, B., Flatt, M., \& Provost, J. (1993). PsyScope: A new graphic interactive environment for designing psychology experiments. Behavioral Research Methods, Instruments, and Computers, 25, 257-271.

Conturo, T. E., McKinstry, R. C., Akbudak, E., Snyder, A. Z., Yang, T. Z., \& Raichle, M. E. (1996). Sensitivity optimization and experimental design in functional magnetic resonance imaging. Society for Neuroscience Abstracts, 22, 7.

Craik, F. I. M., \& Lockhart, R. S. (1972). Levels of processing: A framework for memory research. Journal of Verbal Learning and Verbal Bebavior, 4, 671-684.

Demb, H. B., Desmond, J. E., Wagner, A. D., Vaidya, C. J., Glover, G. H., \& Gabrieli, J. D. E. (1995). Semantic encoding and retrieval in the left inferior prefrontal cortex: A functional MRI study of task difficulty and process specificity. Journal of Neuroscience, 15, 5870-5878.

Desgranges, B., Baron, J.-C., \& Eustache, F. (1998). The functional neuroanatomy of episodic memory: The role of the frontal lobes, the hippocampal formation, and other areas. Neuroimage, 8, 198-213.

Fletcher, P. C., Frith, C. D., Grasby, P. M., Shallice, T., Frackowiak, R. S. J., \& Dolan, R. J. (1995). Brain systems for encoding and retrieving auditory-verbal memory: An in vivo study in humans. Brain, 118, 401-416.

Fletcher, P. C., Frith, C. D., \& Rugg, M. D. (1997). The functional neuroanatomy of episodic memory. Trends in Neurosciences, 20, 213-218.

Friston, K. J., Jezzard, P., \& Turner, R. (1994). Analysis of functional MRI time-series. Human Brain Mapping, 1, 153171.

Gazzaniga, M. S., Bogen, J. E., \& Sperry, R. W. (1962). Some functional effects of sectioning the cerebral commissures in man. Proceedings of the National Academy of Sciences USA, 48, 1765-1769.

Gazzaniga, M. S., \& Smylie, C. S. (1983). Facial recognition and brain asymmetries: Clues to underlying mechanisms. Annals of Neurology 13, 536-540.

Glover, J. A. (1989). The 'testing' phenomemon: Not gone but nearly forgotten. Journal of Educational Psychology 81, 329-399.

Grady, C. L., McIntosh, A. R., Horwitz, B., Maisog, J. M., Ungerleider, L. G., Mentis, M. J., Pietrini, P., Schapiro, M. B., \& Haxby, J. V. (1995). Age-related reductions in human recognition memory due to impaired encoding. Science, 269, 218-221.

Haxby, J. V., Ungerleider, L. G., Horwitz, B., Maison, J. M., 
Rapoport, S. I., \& Grady, C. L. (1996). Face encoding and recognition in the human brain. Proceedings of the $\mathrm{Na}$ tional Academv of Sciences USA, 93, 922-927.

Kapur, S., Craik, F. I. M., Jones, C., Brown, G. M., Houle, S., \& Tulving, E. (1995). Functional role of the prefrontal cortex in retrieval of memories: A PET study. NeuroReport, 6, 1880-1884

Kapur, S., Craik, F. I. M., Tulving, E., Wilson, A. A., Houle, S., \& Brown, G. M. (1994). Neuroanatomical correlates of encoding in episodic memory: Levels of processing effect. Proceedings of the National Academv of Sciences. USA, 91, 2008-2011.

Kelley, W. M., Buckner, R. L., Miezin, F. M., Cohen, N. J., Raichle, M. E., \& Petersen, S. E. (1999). Encoding of famous and nonfamous faces using fMRI. Society for Neuroscience Abstracts, 24, 760.

Kelley, W. M., Buckner, R. L., \& Petersen, S. E. (1998). Response to Nyberg et al. Trends in Cognitive Sciences, 2, 421.

Kelley, W. M., Miezen, F. M., McDermott, K. B., Buckner, R. L., Raichle, M. E., Cohen, N. J., Ollinger, J. M., Akbudak, E., Conturo, T. E., Snyder, A. Z., \& Petersen, S. E. (1998). Hemispheric asymmetry for verbal and nonverbal memory encoding in human dorsal frontal cortex. Neuron, 20, 927936.

Klatzky, R. L., \& Atkinson, R. C. (1971). Specialization of the cerebral hemispheres in scanning for information in shortterm memory. Perception \& Psychophysics, 10, 335-338

MacLeod, A. K., Buckner, R. L., Miezin, F. M., Petersen, S. E., \& Raichle, M. E. (1998). Right prefrontal cortex activation during semantic monitoring and working memory. Neuroimage, 7, 41-48.

McDermott, K. B., Ojemann, J. G., Petersen, S. E., Ollinger, J. M., Snyder, A. Z., Akbudak, E., Conturo, T. E., \& Raichle, M. E. (in press). Direct comparison of episodic encoding and retrieval of words: An event-related fMRI study. Memory.

Milner, B. (1971). Interhemispheric differences in the localization of psychological processes in man. British Medical Bulletin, 27, 272-277.

Mintun, M. A., Fox, P. T., \& Raichle, M. E. (1989). A highly accurate method of localizing regions of neuronal activity in the human brain with positron emission tomography. Journal of Cerebral Blood Flow and Metabolism, 9, 96-103.

Nolde, S. F., Johnson, M. K., \& Raye, C. L. (1998). The role of prefrontal cortex during tests of episodic memory. Trends in Cognitive Sciences, 2, 399-406.

Nyberg, L., Cabeza, R., \& Tulving, E. (1996). PET studies of encoding and retrieval: The HERA model. Psychonomic Bulletin \& Review, 3, 135-148.

Nyberg, L., Cabeza, R., \& Tulving, E. (1998). Asymmetric frontal activation during episodic memory: What kind of specificity? Trends in Cognitive Sciences, 2, 419-420.

Nyberg, L., Tulving, E., Habib, R., Nilsson, L-G., Kapur, S., Houle, S., Cabeza, R., \& McIntosh, A. R. (1995). Functional brain maps of retrieval mode and recovery of episodic information. NeuroReport, 7, 249-252.

Ojemann, J. G., Akbudak, E., Snyder, A. Z., McKinstry, R. C., Raichle, M. E., \& Conturo, T. E. (1997). Anatomic localization and quantitative analysis of gradient refocused echoplanar fMRI susceptibility artifacts. Neuroimage, 6 , 156-167.

Ollinger, J. M. (1997). Correcting for multiple comparisons in fMRI activation studies with region-size dependent thresh- olds. Proceedings of the International Society for Magnetic Resonance in Medicine Abstracts, 1672

Paivio, A. (1971). Imagery and verbal processes. New York: Holt, Rinehart, \& Winston.

Petrides, M., Alivisatos, B., \& Evans, A. C. (1995). Functional activation of the human ventrolateral frontal cortex during mnemonic retrieval of verbal information. Proceedings of the National Academy of Sciences USA, 92, 5803-5807.

Poldrack, R. A., \& Gabrieli, J. D. E. (1998). Memory and the brain: What's right and what's left? Cell, 93, 1091-1093.

Rugg, M. D., Fletcher, P. C., Frith, C. D., Frackowiak, R. S. J., \& Dolan, R. J. (1996). Differential activation of the prefrontal cortex in successful and unsuccessful memory retrieval. Brain, 119, 2073-2083.

Schacter, D. L., Alpert, N. M., Savage, C. R., Rauch, S. L., \& Albert, M. S. (1996). Conscious recollection and the human hippocampal formation: Evidence from positron emission tomography. Proceedings of the National Academy of Science, 93, 321-325.

Schacter, D. L., Buckner, R. L., Koutstaal, W., Dale, A. M., \& Rosen, B. R. (1997). Late onset of anterior prefrontal activity during true and false recognition: An event-related fMRI study. Neuroimage, 6, 259-269.

Sergent, J. (1983). The role of input in visual hemispheric asymmetries. Psychological Bulletin, 93, 481-514.

Snodgrass, J. G., \& Vanderwart, M. (1980). A standardized set of 260 pictures: Norms for name agreement, image agreement, familarity, and visual complexity. Journal of Experimental Psychology: Human Learning and Memorv, 6, 174-215.

Snyder, A. Z. (1996). Difference image versus ratio image error function forms in PET-PET realignment. In D. Bailey \& T. Jones (Eds.), Quantification of brain function using PET (pp. 131-137). San Diego: Academic Press.

Squire, L. R., Ojemann, J. G., Miezin, F. M., Petersen, S. E., Videen, T. O., \& Raichle, M. E. (1992). Activation of the hippocampus in normal humans: A functional anatomical study of memory. Proceedings of the National Academy of Sciences of the USA, 89, 1837-1841.

Talairach, J., \& Tournoux, P. (1988). Co-planar stereotaxic atlas of the buman brain. New York: Thieme.

Tulving, E. (1983). Elements of episodic memory. New York: Oxford University Press.

Tulving, E., Kapur, S., Craik, F. I. M., Markowitsch, H. J., \& Houle, S. (1994). Hemispheric encoding/retrieval asymmetry in episodic memory: Positron emission tomography findings. Proceedings of the National Academv of Sciences USA, 91, 2016-2020.

Wagner, A. D., Desmond, J. E., Glover, G. H., \& Gabrieli, J. D. E. (1998). Prefrontal cortex and recognition memory: fMRI evidence for context-dependent retrieval processes. Brain, 121, 1985-2002.

Wagner, A. D., Poldrack, R. A., Eldridge, L. L., Desmond, J. E., Glover, G. H., \& Gabrieli, J. D. E. (1998). Material-specific lateralization of prefrontal activation during episodic encoding and retrieval. NeuroReport, 9, 3711-3717.

Wagner, A. D., Schacter, D. L., Rotte, M., Koutstaal, W., Maril, A., Dale, A., Rosen, B., \& Buckner, R. L. (1998). Building memories: Remembering and forgetting of verbal experiences as predicted by brain activity. Science, 281, 11881191.

Worsley, K. J., \& Friston, K. J. (1995). Analysis of fMRI time-series revisited-again. Neuroimage, 2, 173-182. 


\section{This article has been cited by:}

1. Giuseppina Rametti, Carme Junqué, Pere Vendrell, Rosa Catalán, Rafael Penadés, Nuria Bargalló, Miguel Bernardo. 2009. Hippocampal underactivation in an fMRI study of word and face memory recognition in schizophrenia. European Archives of Psychiatry and Clinical Neuroscience 259:4, 203-211. [CrossRef]

2. Nozomi Akanuma, Laurence J. Reed, Paul K. Marsden, Jozeph Jarosz, Naoto Adachi, William A. Hallett, Gonzalo Alarcón, Robin G. Morris, Michael Koutroumanidis. 2009. Hemisphere-specific Episodic Memory Networks in the Human Brain: A Correlation Study between Intracarotid Amobarbital Test and [18F]FDG-PETHemisphere-specific Episodic Memory Networks in the Human Brain: A Correlation Study between Intracarotid Amobarbital Test and [18F]FDG-PET. Journal of Cognitive Neuroscience 21:3, 605-622. [Abstract] [Full Text] [PDF] [PDF Plus]

3. Hiroki M. Morimoto, Satoshi Hirose, Junichi Chikazoe, Koji Jimura, Tomoki Asari, Ken-ichiro Yamashita, Yasushi Miyashita, Seiki Konishi. 2008. On Verbal/Nonverbal Modality Dependence of Left and Right Inferior Prefrontal Activation during Performance of Flanker Interference TaskOn Verbal/Nonverbal Modality Dependence of Left and Right Inferior Prefrontal Activation during Performance of Flanker Interference Task. Journal of Cognitive Neuroscience 20:11, 2006-2014. [Abstract] [PDF] [PDF Plus]

4. M. Nishida, J. Pearsall, R. L. Buckner, M. P. Walker. 2008. REM Sleep, Prefrontal Theta, and the Consolidation of Human Emotional Memory. Cerebral Cortex 19:5, 1158-1166. [CrossRef]

5. Tilo Kircher, Susanne Weis, Dirk Leube, Katrin Freymann, Michael Erb, Frank Jessen, Wolfgang Grodd, Reinhard Heun, Sören Krach. 2008. Anterior hippocampus orchestrates successful encoding and retrieval of non-relational memory: an event-related fMRI study. European Archives of Psychiatry and Clinical Neuroscience 258:6, 363-372. [CrossRef]

6. Leun J. Otten, Josefin Sveen, Angela H. Quayle. 2007. Distinct Patterns of Neural Activity during Memory Formation of Nonwords versus WordsDistinct Patterns of Neural Activity during Memory Formation of Nonwords versus Words. Journal of Cognitive Neuroscience 19:11, 1776-1789. [Abstract] [PDF] [PDF Plus]

7. Michael J. Wenger, James T. Townsend. 2006. On the Costs and Benefits of Faces and Words: Process Characteristics of Feature Search in Highly Meaningful Stimuli. Journal of Experimental Psychology: Human Perception and Performance 32:3, 755-779. [CrossRef]

8. Jason P. Mitchell , Chad S. Dodson , Daniel L. Schacter . 2005. fMRI Evidence for the Role of Recollection in Suppressing Misattribution Errors: The Illusory Truth EffectfMRI Evidence for the Role of Recollection in Suppressing Misattribution Errors: The Illusory Truth Effect. Journal of Cognitive Neuroscience 17:5, 800-810. [Abstract] [PDF] [PDF Plus]

9. Roxane J. Itier, Margot J. Taylor. 2004. Effects of repetition and configural changes on the development of face recognition processes. Developmental Science 7:4, 469-487. [CrossRef]

10. Roxane J. Itier, Margot J. Taylor, Nancy J. Lobaugh. 2004. Spatiotemporal analysis of event-related potentials to upright, inverted, and contrast-reversed faces: Effects on encoding and recognition. Psychophysiology 41:4, 643-653. [CrossRef]

11. Charan Ranganath . 2004. The 3-D Prefrontal Cortex: Hemispheric Asymmetries in Prefrontal Activity and Their Relation to Memory Retrieval ProcessesThe 3-D Prefrontal Cortex: Hemispheric Asymmetries in Prefrontal Activity and Their Relation to Memory Retrieval Processes. Journal of Cognitive Neuroscience 16:6, 903-907. [Abstract] [PDF] [PDF Plus]

12. Sander M. Daselaar, Dick J. Veltman, Menno P. Witter. 2004. Common pathway in the medial temporal lobe for storage and recovery of words as revealed by event-related functional MRI. Hippocampus 14:2, 163-169. [CrossRef]

13. Gagan S. Wig, Michael B. Miller, Alan Kingstone, William M. Kelley. 2004. Separable Routes to Human Memory Formation: Dissociating Task and Material Contributions in the Prefrontal CortexSeparable Routes to Human Memory Formation: Dissociating Task and Material Contributions in the Prefrontal Cortex. Journal of Cognitive Neuroscience 16:1, 139-148. [Abstract] [PDF] [PDF Plus]

14. Steven Z. Rapcsak. 2003. Face memory and its disorders. Current Neurology and Neuroscience Reports 3:6, 494-501. [CrossRef]

15. Roberto Cabeza , Jill K. Locantore , Nicole D. Anderson . 2003. Lateralization of Prefrontal Activity during Episodic Memory Retrieval: Evidence for the Production-Monitoring HypothesisLateralization of Prefrontal Activity during Episodic Memory Retrieval: Evidence for the Production-Monitoring Hypothesis. Journal of Cognitive Neuroscience 15:2, 249-259. [Abstract] [PDF] [PDF Plus]

16. Ela I. Olivares , Jaime Iglesias , Socorro Rodríguez-Holguín . 2003. Long-Latency ERPs and Recognition of Facial IdentityLong-Latency ERPs and Recognition of Facial Identity. Journal of Cognitive Neuroscience 15:1, 136-151. [Abstract] [PDF] [PDF Plus] 
17. Takashi Tsukiura, Toshikatsu Fujii, Toshimitsu Takahashi, Ruiting Xiao, Motoaki Sugiura, Jiro Okuda, Toshio Iijima, Atsushi Yamadori. 2003. Medial temporal lobe activation during context-dependent relational processes in episodic retrieval: An fMRI study. Human Brain Mapping 17:4, 203-213. [CrossRef]

18. W. M. Kelley , J. G. Ojemann , R. D. Wetzel , C. P. Derdeyn , C. J. Moran , D. T. Cross , J. L. Dowling, J. W. Miller , S. E. Petersen . 2002. Wada Testing Reveals Frontal Lateralization for the Memorization of Words and FacesWada Testing Reveals Frontal Lateralization for the Memorization of Words and Faces. Journal of Cognitive Neuroscience 14:1, 116-125. [Abstract] [PDF] [PDF Plus]

19. Carol Armstrong, Christianne Stern, Benjamin Corn. 2001. Memory Performance Used to Detect Radiation Effects on Cognitive Functioning. Applied Neuropsychology 8:3, 129-139. [CrossRef]

20. Jon S. Simons, Kim S. Graham, Adrian M. Owen, Karalyn Patterson, John R. Hodges. 2001. Perceptual and Semantic Components of Memory for Objects and Faces: A PET StudyPerceptual and Semantic Components of Memory for Objects and Faces: A PET Study. Journal of Cognitive Neuroscience 13:4, 430-443. [Abstract] [PDF] [PDF Plus]

21. Kathleen B. McDermott, Todd C. Jones, Steven E. Petersen, Sarah K. Lageman, Henry L. Roediger, III . 2000. Retrieval Success is Accompanied by Enhanced Activation in Anterior Prefrontal Cortex During Recognition Memory: An Event-Related fMRI StudyRetrieval Success is Accompanied by Enhanced Activation in Anterior Prefrontal Cortex During Recognition Memory: An Event-Related fMRI Study. Journal of Cognitive Neuroscience 12:6, 965-976. [Abstract] [PDF] [PDF Plus]

22. Roberto Cabeza, Lars Nyberg. 2000. Current Opinion in Neurology 13:4, 415-421. [CrossRef]

23. 2000. Current awareness in NMR in biomedicine. NMR in Biomedicine 13:3, 166-171. [CrossRef] 